\title{
The influence of the nocebo effect in clinical trials
}

This article was published in the following Dove Press journal:

Open Access Journal of Clinical Trials

2 November 2012

Number of times this article has been viewed

\section{Luana Collocal,2,3}

'National Center for Complementary and Alternative Medicine, National Institutes of Health, Bethesda, MD, USA; ${ }^{2}$ National Institute of Mental Health, National Institutes of Health, Bethesda, MD, USA; ${ }^{3}$ Department of Bioethics, The Clinical Center, National Institutes of Health, Bethesda, MD, USA
Correspondence: Luana Colloca, Building 10, Room ICI54, Bethesda, MD 20892-II56, USA

$\mathrm{Tel}+|30| 43587 \mid 5$

Fax +I 3014960760

Email luana.colloca@nih.gov
Abstract: Neurobiological and clinical studies have shown that learning mechanisms and expectations of benefits powerfully affect the brain, mind, and body, with the potential of relieving many symptoms during the course of daily clinical practice. Playing the role of antagonist is the "nocebo effect," which results from negative expectations derived from one's beliefs, previous experiences, and his or her clinical encounters that produce negative effects. Research on the nocebo effect indicates that information disclosure and the manner in which information is delivered can contribute to these adverse effects. In this article, we review neurobiological and medical studies relating to the nocebo effect, as these findings are important for the methodology of clinical trials.

Keywords: adverse events, clinical research, communication, expectation, nocebo effects, side effects

\section{Introduction}

In randomized placebo-controlled clinical trials, patients receiving placebos often report side effects that are similar to those experienced by patients receiving study treatments. Kennedy ${ }^{1}$ as well as Kissel and Barrucand ${ }^{2}$ introduced the term 'nocebo,' which is a phenomenon that produces a negative effect as a result of negative expectations or prior experiences; this is distinguished from beneficial aspects of the 'placebo' effect, whereby positive effects occur based on one's expectations or prior experiences. Nocebo effects are attributable to the communication of potential adverse effects, memories of past unsuccessful therapeutic experiences, and negative psychosocial contexts and clinical encounters. ${ }^{3,4}$ For decades, these observations were dismissed as purely psychological; however, new research indicates that nocebo effects can cause real biological changes, a finding that is transforming how medicine is practiced.

\section{Psychological mechanisms underlying nocebo effects}

Verbal communication, health care providers' behavior, environmental cues, and the presence of medical devices may induce negative expectations among research subjects and patients, which can lead to adverse effects. As is the case with placebo analgesia, pain has become the best model used to investigate the psychological and neurobiological mechanisms of the nocebo effect. ${ }^{5,6}$ Behaviorally, while placebos work to relieve pain and other symptoms, nocebos can strongly increase one's experience of pain and result in many other conditions. ${ }^{7}$ 
In 1981, Schweiger and Parducci introduced a model to study the influences of negative verbal suggestions on localized pain in healthy subjects. The subjects in their study were deceptively informed that an electrical current was going to pass through their heads. Informing subjects about the possibility of experiencing pain induced the anticipated side effect, suggesting that expectations driven by verbal anticipation may affect brain and body responses. ${ }^{8}$ These pioneering observations have been recently confirmed healthy subjects experienced headaches when informed that a radiofrequency passing through their heads could cause pain. As expected, the exposure to the radiofrequency was false, and the participants reported experiencing discomfort and headaches merely because of verbally-induced expectations. ${ }^{9,10}$

Expectations can be created in the experimental setting by verbally suggesting that something negative will happen; furthermore, classical conditioning via prior exposure to deterioration of symptoms can also elicit nocebo effects. Both of these types of nocebo manipulations are able to induce the worsening of symptoms in healthy participants. What is extraordinary is the fact that verbally-induced expectations produce nocebo effects that are comparable in magnitude to those induced by actual experiences that have occurred as a result of conditioning. This has been observed in the context of somatosensory perception, painful experiences, and motor performance. ${ }^{11-13}$ In the context of somatosensory perception, ${ }^{11}$ painful experiences, ${ }^{11,12}$ and motor performance. ${ }^{13}$

Colloca et $\mathrm{a}^{11}$ studied the influences of verbal suggestions and conditioning on pain and tactile perception. Nocebo suggestions were given to healthy volunteers before the administration of either tactile or low-intensity electrical stimuli that induced pain among the participants. The nocebo manipulation was also carried out after a preconditioning session in which two different conditioned visual stimuli were actually associated either with pain or without pain. The magnitude of the nocebo hyperalgesic effects following either verbal suggestions alone or the conditioning procedure were the same, with no significant differences noted. ${ }^{11}$ These findings have been recently confirmed and expanded upon by Van Laarhoven et al, who found that verbally-induced expectations of high levels of pain and itchiness resulted in higher levels of these two sensations, with more pronounced effects noted in itchiness than for pain. ${ }^{12}$ Verbal anticipation of increased load and actual prior experience of load increase similarly impaired physical performance and endurance. ${ }^{13}$
Remarkably, gender effects modulate conditioned and verbally-induced nocebo effects. Healthy men and women were investigated for verbally-induced and conditioned nocebo effects in a model of nausea and vomiting using a rotation procedure. On days 1 and 2, subjects in the conditioning group received a strong oral stimulus prior to rotation; subjects in the control group received the stimulus 12 hours after the rotations. On day 3, all subjects received the stimulus prior to rotation. Another group of healthy subjects were rotated only five times for 1 minute, and received the same oral stimulus while being told that the symptoms might worsen with the stimulus. The negative conditioning reduced the rotation tolerance and significantly increased participants' reported symptom ratings. In this model of nausea, women appeared to be more negatively affected by the conditioning procedure, while verbal suggestions appeared to influence men's symptoms to a greater degree. ${ }^{13}$

Humans are also able to experience negative outcomes based on the observation of others; therefore, social learning represents another mechanism involved in the formation of placebo and nocebo effects. For example, Mazzoni et al studied the effects of observation in a model of mass psychogenic illness. ${ }^{14}$ Healthy subjects were asked to inhale a sample of normal air; however, they were told that the sample contained a suspected environmental toxin known to cause headaches, nausea, itchy skin, and drowsiness. Subsequently, half of the participants observed an actor inhale and display the four expected symptoms. Participants who observed another person become ill were more likely to report experiencing the expected symptoms. ${ }^{14}$ These findings suggest the importance of social observations in supporting mass psychogenic illnesses and the potential implications of nocebo effects for public health. ${ }^{15}$

\section{Imaging nocebo reactions}

Imaging studies have indicated that nocebo effects are associated with specific localized brain changes, along with a deactivation of the dopaminergic and opioidergic systems. For example, Koyama et al demonstrated that expectations heavily shape people's perceptions and evaluations of incoming sensory information. By contrasting the patterns of brain activation related to the intensity of expected pain and actual experienced pain, they found that the magnitude of expected pain increased under negative suggestions. These changes occurred in the thalamus, insula, prefrontal cortex, anterior cingulate cortex, and other brain regions, suggesting that a 'mental representation' of an impending sensory event can significantly shape neural processes that underlie the formulation of the actual sensory experience. ${ }^{16}$ 
Keltner et al ${ }^{17}$ conditioned healthy subjects to one of two noxious thermal stimuli, which varied in terms of intensity (either high or low). Visual cues were presented just prior to and during application of the noxious thermal stimulus. The pain intensities were set at exactly the same level, but subjects reported experiencing significantly higher levels of pain when the noxious stimulus was preceded by the high-intensity visual cue. By comparing the activations produced by the two visual cues that were presented with the high-intensity noxious thermal stimulus, there were significant differences noted in the ipsilateral caudal anterior cingulate cortex, the head of the caudate, cerebellum, and the contralateral nucleus cuneiformis. Negative expectancies activated a distinct modulatory network that converged with afferent nociceptive input in the nucleus cuneiformis. ${ }^{17}$ Scott et al found that healthy subjects who reacted to placebo administration with hyperalgesic nocebo responses showed decreased activation across the mesolimbic dopaminergic system in the ventral basal ganglia, as well as in the endogenous opioid system in the rostral and subgenual anterior cingulate cortex, orbitofrontal cortex, anterior and posterior insulae, medial thalamus, nucleus accumbens, amygdala, and periaqueductal area. ${ }^{18}$

Interestingly, the effects of negative expectations interfere with the analgesic efficacy of remifentanil, a $\mu$ opioid agonist. While positive treatment expectations substantially enhanced (doubled) the analgesic benefit of remifentanil, negative expectations abolished the brain analgesic effect and the subjective pain reports. These subjective changes in drug effectiveness and pain experience were substantiated by significant modifications in the neural activity of brain regions involved with the coding of pain intensity. The negative effects correlated with activity in the hippocampus. ${ }^{19}$ Overall, these brain-imaging studies provided evidence that verbally-induced and conditioned expectations strongly shape an individual's perceptions of pain, and they can also affect the therapeutic efficacy of painkillers among individuals.

\section{Nocebo effects can shape drug actions}

Interestingly, verbally-induced expectations can also interfere with the response to drugs in a paradoxical manner. The examples below show how active drugs administered using contradictory instructions can result in an impairment of outcomes. For instance, asthmatic patients reported reversed responses to bronchoconstrictors that were described as bronchodilators, and vice versa. ${ }^{20}$ Similarly, healthy subjects who believed that they were given a stimulant reported experiencing increased muscle tension, when in actuality they were receiving a muscle relaxant. ${ }^{21}$ Moreover, when participants receive verbal suggestions indicating that pain will increase, the effects associated with this anticipation are strong enough to reverse conditioned placebo analgesia following two days of exposure to nonopioid analgesic, ketorolac..$^{22}$ Interestingly, oral administration of an inert substance (talc) along with verbal suggestions of hyperalgesia induced the hyperactivity of the hypothalamic-pituitaryadrenal (HPA) axis, as assessed by levels of adrenocorticotropic hormone and plasma concentrations of cortisol. ${ }^{23,24}$

Both nocebo-induced hyperalgesia and HPA hyperactivity were blocked by diazepam, one of the most widely-used antianxiety benzodiazepines, which suggests that anxiety plays a major role in these effects. In contrast, the administration of the mixed cholecystokinin (CCK) type-A/B receptor antagonist, proglumide, blocked nocebo hyperalgesia completely, but had no effect on HPA hyperactivity, thus suggesting a specific involvement of CCK in the hyperalgesic component (but not in the anxiety component) of the nocebo effect. ${ }^{24}$ Support for this view comes from animal studies in which the CCK antagonist CI-988 has been found to prevent anxiety-induced hyperalgesia, with an observed effect that was similar to that produced by the established anxiolytic, chlordiazepoxide..$^{25}$

\section{Translating nocebo mechanisms in clinical trials}

Nocebo effects are common in patient populations, ${ }^{4}$ although it is difficult to distinguish apparent from true nocebo effects, as the former can be influenced by potential confounding factors. ${ }^{3}$ For example, headaches are a common side effect of antidepressants, and they can be experienced as a result of simply mentioning headaches as a potential side effect of this medication in the informed consent process. In randomized trials, a significant proportion of depressed patients receiving placebos reported experiencing headaches. ${ }^{26,27}$ This scenario could be an apparent nocebo response, as patients might report the same prevalence of headaches if they were in a no-treatment control group and did not receive placebos. The side effects reported by the placebo group may, to some extent, also reflect the natural history of their condition or common symptoms that everyone experiences, rather than expectation-related effects. Nocebo effects can be identified in placebo-controlled trials by including a no-treatment group, which does not receive a placebo or information about the side effects related to a particular treatment or intervention. Additionally, the strategies for 
measuring side effects should be more accurate. ${ }^{28}$ Single ratings, as made by investigators, are often less reliable than patients self-reports, and side effects should be measured through systematic observer assessments and systematic patient self-reports. ${ }^{26}$

\section{Linkage between disclosures and nocebo effects}

Rosenzweig et $\mathrm{al}^{29}$ reviewed the adverse effects spontaneously reported during placebo administration in 109 double-blind placebo-controlled phase 1 pharmacological studies involving 1228 healthy volunteers. All the studies included single versus repeated administration of the placebo ( 5 to 17 days). The adverse effects were assessed by means of the subjects' spontaneous reports to an openended question asking if the subject had anything to report, which were assessed together with observations of the investigators (although this was done without checklists or specific questionnaires). The researchers found an overall incidence of $19 \%$ of adverse effects, which reached a rate of $28 \%$ after repeated dosages. Interestingly, the incidence of adverse effects was higher in elderly subjects (26\%). The most frequent adverse effects were headaches (7\%), drowsiness (5\%), and asthenia (4\%), with some variation that was found to be related to the design and the population used in this study. ${ }^{29}$ It is important to appreciate the study's limitations, particularly the absence of a no-treatment group, which can be useful for identifying confounding factors.

Nocebo effects have been studied in recent systematic reviews of randomized, double-blinded, placebo-controlled studies for headache and migraine treatments. ${ }^{30-32}$ Amanzio et al performed a systematic review of adverse effects of anti-migraine randomized placebo-controlled clinical trials. ${ }^{32}$ The final sample consisted of 69 studies including 56 trials for triptans, 9 trials for anticonvulsants, and 8 trials for nonsteroidal anti-inflammatory drugs. The researchers found a high rate of adverse events in the placebo arms of trials, with the effects noted after taking antimigraine drugs matching those described for real drugs. For example, anticonvulsant placebos produced anorexia, memory difficulties, paresthesia and upper respiratory tract infections - all of which were adverse events reported in the side effect profile of the three classes of antimigraine drugs. ${ }^{32}$ The linkage between reported side effects in the placebo groups and the known side effects of particular drugs suggests genuine nocebo effects that arose from the informed consent process.
Common side effects such as restlessness (34\%), nausea (33\%), anorexia (53\%), and insomnia (79\%) have also been described in patients with advanced cancer (mostly breast cancer) enrolled in randomized double-blind placebo clinical trials for fatigue. ${ }^{33}$ In particular, nausea represents one of the most debilitating and severe side effects among cancer patients, negatively affecting nutrition, adherence to therapy, and quality of life, thus suggesting the importance of better understanding the extent to which these adverse effects might be related to negative expectations and conditioning mechanisms..$^{34,35}$

\section{Nocebo reactions and treatment discontinuation}

Discontinuation and lack of adherence are also common problems in clinical trials and practice, mostly related to the occurrence of adverse events and, likely, nocebo effects. In 1987, Myers et $\mathrm{al}^{36}$ noted that communicating the potential side effects of a given treatment led to participants' subsequent withdrawal from the study. Myers et al retrospectively analyzed the influences that the mere mention of gastrointestinal side effects in consent forms had on patient outcomes across two of three centers involved in a randomized, double-blind, placebo-controlled trial. This study specifically examined the benefit of aspirin, sulfinpyrazone, or both drugs for unstable angina pectoris. The authors found that the inclusion of possible gastrointestinal side effects in the consent forms resulted in a remarkable increase (six-fold) in both patients' reported experiences of gastrointestinal symptoms and consequent patient-initiated cessation of therapy. ${ }^{36,37}$

More recently, Rief et $\mathrm{al}^{28}$ evaluated the occurrence of adverse effects and discontinuation rates in randomized placebo-controlled trials of statin drugs and in populationbased studies. They observed a large degree of variability in patients' experiences of symptoms in the placebo groups across trials, depending on the specific symptom. For example, patients' reported experiences of headaches varied from $0.2 \%$ to $2.7 \%$, and experiences of abdominal pain ranged from $0.9 \%$ to $3.9 \%$ across statin trials performed between 1994 and 2003 among a sample of more than 100 subjects. The rate of adverse events observed in the population-based studies were higher than those observed in randomized placebo-controlled trials, probably because trial participants typically don't represent the population as a whole.

The success rates of prior exposure to conventional treatments have important long-lasting effects. Those who 
experienced ineffective interventions are prone to exhibit or report negative outcomes and adverse events in subsequent treatments. ${ }^{38,39}$ In cross-over and run-in designs, adverse events can occur as a result of treatment sequence and perceived treatment efficacy. A typical nocebo reaction in clinical trials might be evident as the recurrence of symptoms at the end of treatment following drug withdrawal when a placebo is given. In order to overcome such rebound effects, blinded randomized withdrawal strategies can be adopted. ${ }^{40}$

\section{Communicating about adverse events and clinical outcomes}

Most of the studies described above have retrospectively analyzed the occurrence of nocebo effects. More rigorous assessment of nocebo effects has been documented in clinical studies that were specifically designed to investigate the relationship between informing patients about the adverse side effects associated with a specific treatment, and with the occurrence of adverse effects in conditions such as allergies, Parkinson's disease, anxiety, pain, and sexual disorders. For example, nocebo effects have been found among outpatients with adverse drug reactions undergoing an oral drug challenge, indicating that both subjective (itching, nausea, headache, and abdominal pain) and objective (dyspnea, cough, hypotension, tachycardia, erythema, and urticaria) adverse effects can be psychologically-sustained. ${ }^{41,42}$

The influence of patient-doctor communication over clinical outcomes is clearly illustrated by both open and hidden interruption of treatments. ${ }^{43}$ Patients with Parkinson's disease showed a worsening of bradykinesia and velocity of movement when they were informed about the deactivation of deep stimulation of their subthalamic nuclei. ${ }^{22,44}$ According to the open/hidden paradigm, anxiolytics and analgesics were given by means of a computer-controlled infusion pump, and patients were either alerted or not alerted by a provider about the interruption of treatment. Patients openly informed about the interruption of treatment experienced a sudden increase in anxiety and pain, whilst the hidden interruption (controlled by computer) did not induce a worsening, thus suggesting that the mere communication of treatment interruption aggravated patients' symptoms. ${ }^{44}$

The impact of communicating potential adverse side effects has been studied in sexually active patients with benign prostatic hyperplasia (BPH) who received finasteride (5 mg), which was described as a "compound of proven efficacy for the treatment of BPH." Patients were randomized to two different disclosure groups: one group was informed that the medication "may cause erectile dysfunction, decreased libido, problems of ejaculation but these are uncommon"; the other group was not told about these side effects. After 6 and 12 months of follow-up, it was revealed that finasteride administration produced a significantly higher rate of reported sexual problems in those patients who were informed about the possibility of sexual dysfunction (43.6\%) as compared to those among whom the same information was omitted $(15.3 \%){ }^{45}$

Differential effects of pain were also observed when local anesthesia was presented using different descriptions. Healthy, at term, pregnant women requesting epidural analgesia were randomized to either a common description of the pain experience from local anesthesia, "You are going to feel a big bee sting; this is the worst part of the procedure," or a more positive description, "We are going to give you a local anesthetic that will numb the area and you will be comfortable during the procedure." Those women who were informed to expect a bee-like sting during the local anesthetic injection (nocebo group) rated their pain experiences significantly higher than those receiving the procedure in conjunction with a more positive description. Therefore, small changes in the way information is framed really matters. ${ }^{46}$

Overall, these examples illustrate the impact of verbal (and non-verbal) communication on symptom perception. Moreover, it is plausible to think that clinical misdiagnoses represent a circumstance where nocebo reactions might occur. Although well-documented case-reports are lacking, assigning a diagnosis without objective physical evidence may make patients worse, and result in detrimental nocebo effects. $^{27}$

\section{Concluding remarks}

Clinical research and ethics form an inseparable union, and the aforementioned studies on behavioral and brain mechanisms are no different. In the past, the mere use of placebos in medicine has been fraught with stigma, and conjures thoughts of deception and deceit. The nocebo research outlines the need to reconsider the importance of expectancy-related adverse events in clinical trials. ${ }^{47,48}$ It should be evident that verbal instructions are powerful, and can trigger negative brain-mind-body responses. Therefore, it is important to realize that an effective translation of the results from these studies into clinical practice warrants that a clinician's words be carefully selected when describing treatment or intervention options to his or her 
patients. Moreover, a clinician's attitudes towards a given treatment or intervention should be ones that can potentially facilitate the improvement of a patient's symptoms, without unintentionally eliciting untenable nocebo effects. Often, divulging information about treatments is akin to an acrobat performing balancing feats. The examples of concealing information (eg, sexual dysfunction related to taking finasteride for $\mathrm{BPH}$ ) are debatable, as some patients may not agree to undergo the treatment. Clinicians and researchers have an obligation to convey truthful information to patients so that they can make informed decisions in light of their personal preferences and values. An "authorized concealment" approach, whereby patients can consent to receiving information only about the potential serious or irreversible harmful effects, is one prospect worth consideration. ${ }^{49}$

However, there are at least two potential alternatives to "authorized concealment": (1) conveying information by taking advantage of framing strategies; and (2) educating physicians (and researchers) about the reality of the nocebo phenomenon. ${ }^{48}$ There are many examples of different framing strategies that are apparent in many daily circumstances. For instance, a physician who is recommending a drug to a patient may communicate the proportion of patients who experience the most common side effects. Side effects, such as headaches or nausea, may be mentioned merely as a slight possibility. There is also a choice in determining whether and how to communicate the probability that a particular adverse effect will be experienced, as indicated in both quantitative and qualitative studies. ${ }^{50}$ This information can be conveyed by focusing on the minority of patients who experience a particular side effect, or it can be delivered by focusing on the majority of patients who do not experience the side effect. These different ways of framing side effect information can have various impacts on patients' own perceptions of adverse events, ${ }^{51}$ and can also propagate or mitigate the occurrence of nocebo effects. ${ }^{52}$ In addition, the physician (and researchers) may tell the patient that previous research has indicated that some of the side effects can occur merely as a result of telling them about certain side effects.

A variety of studies have investigated the effects of framing information regarding the risks and benefits of interventions on patient decision-making, ${ }^{53}$ but limited clinically-oriented research has considered the impact of informing patients about the nocebo effect as a brain-body interaction. Research on the nocebo effect outlines the importance of framing information in a way that minimizes negative consequences that can occur as a result of alerting patients about potential adverse events and consenting them. Researchers should also consider nocebo reactions and the link between conveying information and occurrence of certain negative outcomes. Researchers have yet to heed Walter Kennedy's thoughts concluding his 1961 article, ${ }^{1}$ where he states: "One wonders how often a useful drug has been discarded because of 'toxic effect' in the first trials which have been due to the accident of there being an appreciable number of nocebo reactors in the test subjects." This doubt is even more relevant today than it was 50 years ago, given that recent advances and brain imaging technologies have increased knowledge of the behavioral and neurobiological bases of the nocebo phenomenon.

\section{Search strategy and selection criteria}

We searched PubMed, the Cochrane library, and Embase databases for reports published in English using the following search terms "nocebo," "nocebo effect," "nocebo response," "adverse events," "side effects," "placebo adverse side effects," "expectation," "conditioning," "observation," "informed consent," and "disclosure." No predefined inclusion or exclusion criteria were used. We included publications from the past 20 years, and also included older publications. We purposely selected publications that were deemed to be most relevant for investigating the clinical and ethical implications of nocebo effects, with emphasis on patientoriented research.

\section{Acknowledgments}

This research was supported by the Intramural Research Program of the National Center for Complementary and Alternative Medicine and the National Institute of Mental Health.

\section{Disclosure}

The opinions expressed are those of the author and do not necessarily reflect the position or policy of the National Institutes of Health, the Public Health Service, or the Department of Health and Human Services. The author has no conflicts of interest to declare.

\section{References}

1. Kennedy WP. The nocebo reaction. Med World. 1961;95:203-205.

2. Kissel P, Barrucand D. Placebos et Effet Placebo en Medecine. [Placebos and placebo effects in medicine]. Paris: Masson; 1964. French.

3. Rief W, Buhlmann U, Wilhelm S, Borkenhagen A, Brahler E. The prevalence of body dysmorphic disorder: a population-based survey. Psychol Med. 2006;36(6):877-885. 
4. Barsky AJ, Saintfort R, Rogers MP, Borus JF. Nonspecific medication side effects and the nocebo phenomenon. JAMA. 2002;287(5): 622-627.

5. Colloca L, Benedetti F. Nocebo hyperalgesia: how anxiety is turned into pain. Curr Opin Anaesthesiol. 2007;20(5):435-439.

6. Colloca L, Miller FG. The nocebo effect and its relevance for clinical practice. Psychosom Med. 2011;73(7):598-603.

7. Benedetti F, Lanotte M, Lopiano L, Colloca L. When words are painful: unraveling the mechanisms of the nocebo effect. Neuroscience. 2007; 147(2):260-271.

8. Schweiger A, Parducci A. Nocebo: the psychologic induction of pain. Pavlov J Biol Sci. 1981;16(3):140-143.

9. Oftedal G, Straume A, Johnsson A, Stovner LJ. Mobile phone headache: a double blind, sham-controlled provocation study. Cephalalgia. 2007;27(5):447-455.

10. Stovner LJ, Oftedal G, Straume A, Johnsson A. Nocebo as headache trigger: evidence from a sham-controlled provocation study with RF fields. Acta Neurol Scand Suppl. 2008;188:67-71.

11. Colloca L, Sigaudo M, Benedetti F. The role of learning in nocebo and placebo effects. Pain. 2008;136(1-2):211-218.

12. RiefW, Henningsen $P$, Hiller W. Classification of somatoform disorders. Am J Psychiatry. 2006;163(4):746-847; author reply 747-748.

13. Klosterhalfen S, Kellermann S, Braun S, et al. Gender and the nocebo response following conditioning and expectancy. J Psychosom Res. 2009;66(4):323-328.

14. Mazzoni G, Foan L, Hyland ME, Kirsch I. The effects of observation and gender on psychogenic symptoms. Health Psychol. 2010;29(2):181-185.

15. Hahn RA. The nocebo phenomenon: concept, evidence, and implications for public health. Prev Med. 1997;26(5 Pt 1):607-611.

16. Koyama T, McHaffie JG, Laurienti PJ, Coghill RC. The subjective experience of pain: where expectations become reality. Proc Natl Acad Sci USA. 2005;102(36):12950-12955.

17. Keltner JR, Furst A, Fan C, Redfern R, Inglis B, Fields HL. Isolating the modulatory effect of expectation on pain transmission: a functional magnetic resonance imaging study. J Neurosci. 2006;26(16):4437-4443.

18. Scott DJ, Stohler CS, Egnatuk CM, Wang H, Koeppe RA, Zubieta JK. Placebo and nocebo effects are defined by opposite opioid and dopaminergic responses. Arch Gen Psychiatry. 2008;65(2):220-231.

19. Bingel U, Wanigasekera V, Wiech K, Ni Mhuircheartaigh R, Lee MC, Ploner M, Tracey I. The effect of treatment expectation on drug efficacy: imaging the analgesic benefit of the opioid remifentanil. Sci Transl Med. 2011 Feb 16;3(70):70ra14.

20. Luparello TJ, Leist N, Lourie CH, Sweet P. The interaction of psychologic stimuli and pharmacologic agents on airway reactivity in asthmatic subjects. Psychosom Med. 1970;32(5):509-513.

21. Flaten MA, Simonsen T, Olsen H. Drug-related information generates placebo and nocebo responses that modify the drug response. Psychosom Med. 1999;61(2):250-255.

22. Benedetti F, Pollo A, Lopiano L, Lanotte M, Vighetti S, Rainero I. Conscious expectation and unconscious conditioning in analgesic, motor, and hormonal placebo/nocebo responses. J Neurosci. 2003;23(10):4315-4323.

23. Benedetti F, Amanzio M, Casadio C, Oliaro A, Maggi G. Blockade of nocebo hyperalgesia by the cholecystokinin antagonist proglumide. Pain. 1997;71(2):135-140.

24. Benedetti F, Amanzio M, Vighetti S, Asteggiano G. The biochemical and neuroendocrine bases of the hyperalgesic nocebo effect. J Neurosci. 2006;26(46):12014-12022.

25. Andre J, Zeau B, Pohl M, Cesselin F, Benoliel JJ, Becker C. Involvement of cholecystokininergic systems in anxiety-induced hyperalgesia in male rats: behavioral and biochemical studies. J Neurosci. 2005;25(35):7896-7904.

26. Rief W, Barsky AJ, Glombiewski JA, Nestoriuc Y, Glaesmer H, Braehler E. Assessing general side effects in clinical trials: reference data from the general population. Pharmacoepidemiol Drug Saf. 2011;20(4):405-415.
27. Kasdan ML, Lewis K, Bruner A, Johnson AL. The nocebo effect: do no harm. J South Orthop Assoc. 1999;8(2):108-113.

28. Rief W, Avorn J, Barsky AJ. Medication-attributed adverse effects in placebo groups: implications for assessment of adverse effects. Arch Intern Med. 2006;166(2):155-160.

29. Rosenzweig P, Brohier S, Zipfel A. The placebo effect in healthy volunteers: influence of experimental conditions on the adverse events profile during phase I studies. Clin Pharmacol Ther. 1993;54(5): $578-583$.

30. Reuter U, Sanchez del Rio M, Carpay JA, Boes CJ, Silberstein SD; for GSK Headache Masters Program. Placebo adverse events in headache trials: headache as an adverse event of placebo. Cephalalgia. 2003;23(7):496-503.

31. Mitsikostas DD, Mantonakis LI, Chalarakis NG. Nocebo is the enemy, not placebo. A meta-analysis of reported side effects after placebo treatment in headaches. Cephalalgia. 2011;31(5):550-561.

32. Amanzio M, Corazzini LL, Vase L, Benedetti F. A systematic review of adverse events in placebo groups of anti-migraine clinical trials. Pain. 2009;146(3):261-269.

33. de la Cruz M, Hui D, Parsons HA, Bruera E. Placebo and nocebo effects in randomized double-blind clinical trials of agents for the therapy for fatigue in patients with advanced cancer. Cancer. 2010;116(3):766-774.

34. Stockhorst U, Wiener JA, Klosterhalfen S, Klosterhalfen W, Aul C, Steingruber HJ. Effects of overshadowing on conditioned nausea in cancer patients: an experimental study. Physiol Behav. 1998;64(5): $743-753$.

35. Colagiuri B, Zachariae R. Patient expectancy and post-chemotherapy nausea: a meta-analysis. Ann Behav Med. 2010;40(1):3-14.

36. Myers MG, Cairns JA, Singer J. The consent form as a possible cause of side effects. Clin Pharmacol Ther. 1987;42(3):250-253.

37. Cairns JA, Gent M, Singer J, et al. Aspirin, sulfinpyrazone, or both in unstable angina. Results of a Canadian multicenter trial. NEngl J Med. 1985;313(22): 1369-1375.

38. Andre-Obadia N, Magnin M, Garcia-Larrea L. On the importance of placebo timing in rTMS studies for pain relief. Pain. 2011;152(6): 1233-1237.

39. Colloca L. Learned placebo analgesia in sequential trials: what are the Pros and Cons? Pain. 2011;152(6):1215-1216.

40. O’Donoghue DP, Dawson AM, Powell-Tuck J, Bown RL, LennardJones JE. Double-blind withdrawal trial of azathioprine as maintenance treatment for Crohn's disease. Lancet. 1978;2(8097):955-957.

41. Lombardi C, Gargioni S, Canonica GW, Passalacqua G. The nocebo effect during oral challenge in subjects with adverse drug reactions. Eur Ann Allergy Clin Immunol. 2008;40(4):138-141.

42. Liccardi G, Senna G, Russo M, et al. Evaluation of the nocebo effect during oral challenge in patients with adverse drug reactions. J Investig Allergol Clin Immunol. 2004;14(2):104-107.

43. Benedetti F, Carlino E, Pollo A. Hidden administration of drugs. Clin Pharmacol Ther. 2011;90(5):651-661.

44. Colloca L, Lopiano L, Lanotte M, Benedetti F. Overt versus covert treatment for pain, anxiety, and Parkinson's disease. Lancet Neurol. 2004;3(11):679-684.

45. Mondaini N, Gontero P, Giubilei G, et al. Finasteride $5 \mathrm{mg}$ and sexual side effects: how many of these are related to a nocebo phenomenon? J Sex Med. 2007;4(6):1708-1712.

46. Varelmann D, Pancaro C, Cappiello EC, Camann WR. Noceboinduced hyperalgesia during local anesthetic injection. Anesth Analg. 2010;110(3):868-870.

47. Brody H, Colloca L, Miller FG. The placebo phenomenon: implications for the ethics of shared decision-making. J Gen Intern Med. 2012;27(6): 739-742.

48. Colloca L, Finniss D. Nocebo effects, patient-clinician communication, and therapeutic outcomes. JAMA. 2012;307(6):567-568.

49. Martin A, Rief W, Klaiberg A, Braehler E. Validity of the Brief Patient Health Questionnaire Mood Scale (PHQ-9) in the general population. Gen Hosp Psychiatry. 2006;28(1):71-77. 
50. Peters E, Hart PS, Fraenkel L. Informing patients: the influence of numeracy, framing, and format of side effect information on risk perceptions. Med Decis Making. 2011;31(3):432-436.

51. Woloshin S, Schwartz LM. Communicating data about the benefits and harms of treatment: a randomized trial. Ann Intern Med. 2011;155(2):87-96.
52. O'Connor AM, Pennie RA, Dales RE. Framing effects on expectations, decisions, and side effects experienced: the case of influenza immunization. J Clin Epidemiol. 1996;49(11):1271-1276.

53. Edwards A, Elwyn G, Covey J, Matthews E, Pill R. Presenting risk information - a review of the effects of "framing" and other manipulations on patient outcomes. J Health Commun. 2001;6(1):61-82.

\section{Publish your work in this journal}

The Open Access Journal of Clinical Trials is an international, peerreviewed, open access journal publishing original research, reports, editorials, reviews and commentaries on all aspects of clinical trial design, management, legal, ethical and regulatory issues, case record form design, data collection, quality assurance and data auditing methodologies. The manuscript management system is completely online and includes a very quick and fair peer-review system, which is all easy to use. Visit http://www.dovepress.com/testimonials.php to read real quotes from published authors.

Submit your manuscript here: http://www.dovepress.com/open-access-journal-of-clinical-trials-journal 